Journal of Case Reports 2018;8(2):147-149

\title{
Accidental Cannulation of Left Superior Intercostal Vein: A Rare Instance of Central Venous Catheter Malpositioning
}

\author{
Srinath Shankar Rathod ${ }^{1}$, Gopal Sharma² \\ ${ }^{1}$ Department of General Surgery, Shalby Hospital, Mohali, Punjab; ${ }^{2}$ Department of General Surgery, University College of Medical \\ Sciences \& Guru Teg Bahadur Hospital, Delhi, India.
}

\section{Corresponding Author:}

Dr. Srinath SR

Email: srinath226@gmail.com

This is an Open Access article distributed under the terms of the Creative Commons Attribution License (creativecommons.org/ licenses/by/3.0).

Received : March 11,2018

Accepted : May 7, 2018

Published : June 10,2018

\begin{abstract}
Background: Central venous catheter (CVC) placement plays an important role in the management of critically ill patients. Complication rates have been reduced with the use of image guidance for their placement. Sometimes, particularly in emergency situation or due to lack of image guidance accidental malpositioning of these catheters can occur further adding to morbidity of the critically ill patients. Case Report: Herein we report a case of CVC placement into left superior intercostal vein during a left sided internal jugular vein (IJV) CVC placement. Conclusion: High index of suspicion along with thorough knowledge of anatomy is required to early detect malposition of central venous catheter.
\end{abstract}

Keywords: Brachiocephalic Veins, Central Venous Catheters, Critical Illness, Jugular Veins.

\section{Introduction}

Central venous catheter (CVC) placement is an important invasive intervention commonly undertaken in critically ill patients for central venous pressure (CVP) measurement, administration of inotropes, blood products, and parenteral nutrition. It is not uncommon to encounter complications during its placement. It is recommended that central venous catheter should be placed under ultrasound guidance. Post-placement, check radiograph is mandatory to confirm proper tip positioning and rule out puncture related complications. Finding poorly functioning $\mathrm{CVC}$ or abnormal course of the catheter on radiography should prompt evaluation. The malpositioned catheter, if found, should be repositioned in order to ensure optimal use and prevent serious complications arising thereof. We report a case of CVC placement into left superior intercostal vein in a young female which was accidentally catheterized during a left sided subclavian CVC placement.

\section{Case Report}

A 22-year-old female, known case of disseminated tuberculosis was referred from a local hospital to our center. There she underwent exploratory laparotomy for perforation of the second part of the duodenum and was referred to our center with shock and bilious discharge from the main wound. She already had right internal jugular vein (IJV) CVC that had to be removed due to infection of the catheter. Thus, the decision to place CVC in internal jugular vein (IJV) on the left side was made and written informed consent from patient's relatives was taken. After thorough aseptic precautions and using ultrasound guidance CVC was placed in left IJV vein. Check radiographs [Fig.1] postCVC placement showed that the left sided IJV was cannulated and CVC was in situ with good back flow and smooth forward flow of fluids except in the distal port. However, next day poor backflow raised the suspicion of malposition of the catheter. The diagnostic confusion regarding malposition 
of the catheter was resolved after contrast dye injection through the CVC which showed [Fig.2] that the catheter had traversed the left superior intercostal vein. The CVC was repositioned and no complication occurred related to CVC.

\section{Discussion}

Accidental insertion of CVC into left superior intercostal vein is a rare occurrence $[1,2]$. Without sonographic or fluoroscopic guidance CVC malposition occurs in about one-third of the cases [3]. However, its frequency is less under image guidance. There have been rare cases of accidental placement of the catheter in the left superior intercostal vein, hemiazygos and accessory hemiazygos vein.

Embryologically, left superior intercostals vein is a remnant of upper part of left cardinal vein. It is formed by the confluence of second to fourth intercostals veins, and in about $75 \%$ of the individuals it communicates with the accessory hemiazygous vein and finally drains into left brachiocephalic vein. Its diameter can range from $1-4 \mathrm{~mm}$ and diameter of more than $4.5 \mathrm{~mm}$ is abnormal [4]. Dilatation of left superior intercostal vein can be due to various causes [5]. Congenital causes of dilatation of left superior intercostals veins include hypoplasia of left brachiocephalic vein and absent inferior vena cava (IVC). Acquired causes include obstruction of great veins (IVC and SVC), portal hypertension and Budd-Chiari syndrome.

On upright postero-anterior chest radiograph, the left superior intercostal vein can be identified in $1.4 \%-9.5 \%$ of healthy people as a small "nipple" lateral to the aortic arch [4]. On plain x-ray of chest dilated left superior intercostals vein could be difficult to differentiate from an aortic aneurysm and enlarged lymph node [4]. In the presence of persistent left superior vena cava (SVC), left superior intercostals vein drains into it. The position of the CVC in the left superior

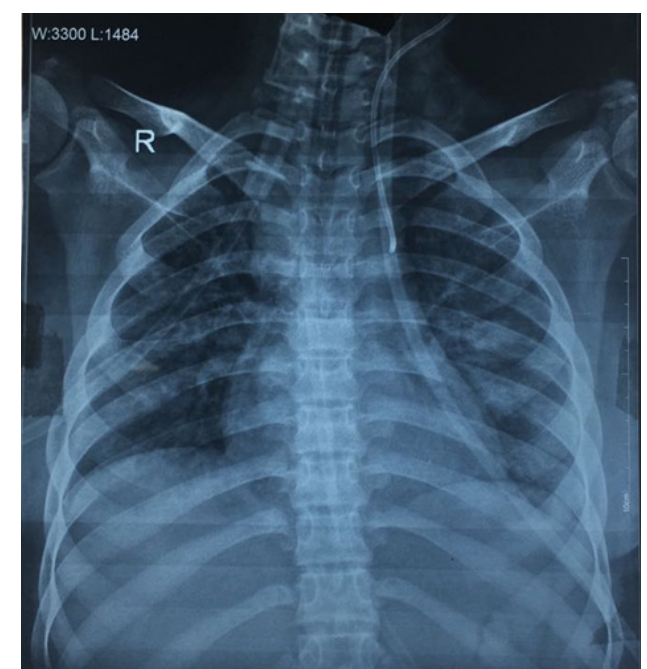

Fig.1: Postero-anterior chest X-ray depicting CVC in left IJV immediate post-insertion.

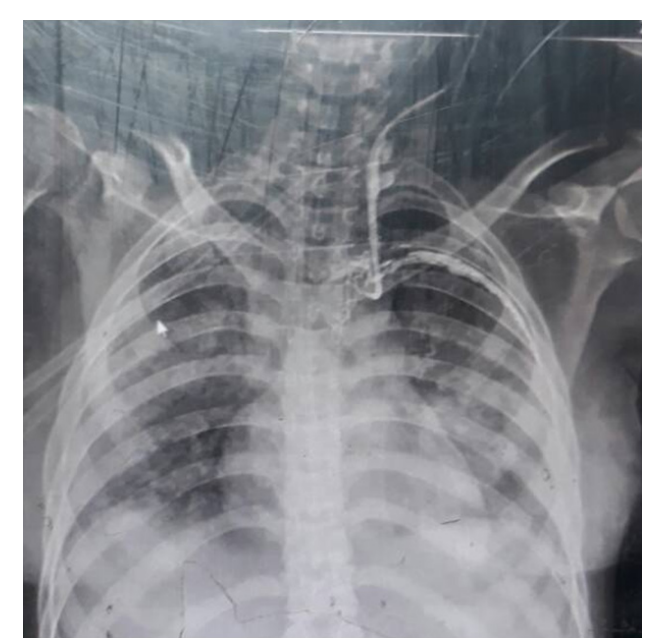

Fig.2: Postero-anterior chest X-ray shows opacification of left superior intercostal vein on contrast injection through CVC.

intercostal vein can be distinguished on chest x-ray from that of the catheter in a persistent left superior vena cava, as the latter descend along left side of the mediastinum without arching laterally over aortic arch [4].

The non-dilated left superior intercostal vein is usually too narrow for the insertion of a CVC. The insertion of the CVC tip into the narrow left superior intercostal vein irritates the 
vessel wall leading to catheter-related infection and thrombosis. Cause for dilated left superior intercostals vein was not further evaluated in this patient as she had no clinical symptomatology to point out any other abnormality. Clinically, accidental malposition should be suspected when there is poor forward or back flow from any of the CVC ports. Post-insertion chest radiograph is mandatory to check tip position and identifying procedure related complications. Whenever there is clinical and radiological suspicion of malposition further investigation is warranted either in form of a lateral view skiagram or cross sectional imaging. Dynamic radiograph while injecting small volume contrast into central venous catheter port can help in delineating venous anatomy and confirm tip location. Padovan et al. [6] and Vahid et al. [7] used computed tomography (CT) to confirm the malposition of the catheter into left superior intercostals vein. Computed tomography (CT) may be of use to confirm the tip location, however, routine use cannot be recommended for confirming catheter tip due to concerns of high radiation exposure. Instead, dynamic radiograph using the available malpositioned catheter and small amount of contrast appears to be a reasonable option. Volume of contrast used may not have much renal consequences in most of the cases and information obtained is valuable as compared to amount of radiation required for $\mathrm{CT}$ scan.

\section{Conclusion}

Dynamic radiograph with approximately $15 \mathrm{ml}$ of contrast into the central vein catheter can be a valuable tool in evaluation of the CVC position, especially in patients in whom diagnostic contrast venography is contraindicated due to renal failure. However thorough knowledge of normal and anomalous venous anatomy is required for the interpretation of the central vein catheter position.

Contributors: SSR: manuscript writing, patient management; GS: manuscript editing, patient management. SSR will act as guarantor. Both authors approved the final version of this manuscript.

Funding: None; Competing interests: None stated.

\section{References}

1. Shapiro MJ, Allen HM, Talpos GB. Internal thoracic vein cannulation as a complication of central venous catheterization. Am Surg. 1982;48:408e11.

2. Sarnak MJ, Levey AS. Placement of an internal jugular dialysis catheter into superior intercostal vein. Nephrol Dial Transplant. 1999;14:2028-2029.

3. Godwin JD, Chen JTT. Thoracic venous anatomy. AJR Am J Roentgenol. 1985;147:674-684.

4. Friedman AC, Chambers E, Sprayregen S. The normal and abnormal left superior intercostal vein. AJR Am J Roentgenol. 1978;131:599-602.

5. Pagniez B, Denies JD, Dupus C, Remy J. The left superior intercostal vein. J Radiol Electrol. 1975;56:285298.

6. Padovan RS, Paar MH, Aurer I.(Mis)placed central venous catheter in the left superior intercostal vein. Radiol Oncol. 2011;45:27-30.

7. Vahid B, Kotiah S, Marik P. Malposition of central venous catheter in left superior intercostal vein in a patient with superior vena cava syndrome. Radiography. 2007;13:307-309. 